\title{
Manual Liquid Based Cytology for Pap Smear Preparation and HPV Detection by PCR in Pakistan
}

\author{
Shehla Akbar ${ }^{1}$, Shgufta Nasir Pervez ${ }^{2}$, Walayat Shah ${ }^{1 *}$
}

\begin{abstract}
This study was conducted on female patients with different gynecological problems attending the gynecology out-patient departments of two tertiary care hospitals in Peshawar city of Khyber Pakhtunkhwa, Pakistan between August 2012 and October 2013. The 200 patients had an age range of 21-65 years. Smears were taken with cervical brushes and preserved in preservative medium and processed for manual liquid based cytology (MLBC) for Pap staining. Out of 200 collected samples, 30 samples were found inadequate on cytology. Of the remaining 170 samples, $164(96.47 \%)$ were normal, $5(2.94 \%)$ were of atypical squamous cells of unknown significance (ASCUS) and $1(\mathbf{0 . 6 \%})$ was of high grade squamous intraepithelial lesion (HSIL). On PCR all the samples were positive for beta globin gene fragment including those reported inadequate on cytology. Out of the 5 ASCUS samples, 2 samples were positive for HPV, one each for HPV 16 and HPV 18, and the rest of the 3 samples were negative for HPV DNA. The 1 sample of HSIL was positive for HPV 16 on PCR. Out of 164 normal samples on cytology, only 1 sample was HPV 16 positive. So overall, $4(2 \%)$ out of 200 samples were positive for HPV DNA, where 3 were HPV $16(1.5 \%)$, and 1 was HPV $18(0.5 \%)$ positive, and thus the ratio of infection with of HPV 16 to HPV 18 was 3:1 in the general population. In conclusion, PCR based HPV detection is a more sensitive method for screening of HPV infection than cytology as sample inadequacy does not affect the results. However, it can be combined with cytology methods in a HPV positive female to achieve the maximum results.
\end{abstract}

Keywords: Cervical cancer - HPV - Papanicolaou stain - manual liquid based cytology - Pakistan

Asian Pac J Cancer Prev, 16 (2), 579-583

\section{Introduction}

Cervical cancer is the third most commonly occurring cancer (Mathers et al., 2010) among women Worldwide and is one of the major public health encumbrances. Human papillomavirus (HPV) is the main culprit (zur Hausen, 1999) in the causation of cervical cancer. It is anticipated that at some point in their lives, around $79 \%$ of women, globally, get HPV infection (Koutsky, 1997). The rates of attaining an HPV infection peak soon after the age when most young women become sexually active (Dunne and Markowitz, 2006). Unprotected sexual contact is the main route of transmission (Garnett et al., 2002) of genital HPV with a high probability of transmission estimated to be $50-80 \%$.The first step which is essential for cervical cancer to develop is infection of the cervix by one of the high risk types of HPV carrying oncogenic potential (Schiffmann, 2007).

The facts gathered so far about cervical cancer shows that this can be controlled through primary as well as secondary preventive measures. Primary prevention relies on the general awareness of women about risk factors and requisite changes in life style which is a main question in the developing countries where educational level of the women is yet to progress considerably (Nandini, 2012). Therefore, in these countries the center of attention is secondary prevention through early cytologic detection and conventional Pap smear cytology happens to be the foundation of it. However, its widespread use has not been possible in these countries (Nandini, 2012) due to shortage of resources, technical staff and other amenities. Also its sensitivity is said to reduce to less than $50 \%$ when there is presence of obscuring blood, inflammatory cells or broad areas of overlapping epithelial cells, even though the rate of invasive cervical cancer is decreased through this method of screening. Around 30\% cases (ACOG Committee, 2009) reported negative on Pap cytology test recently done turned out to have cervical cancer.

Liquid based cytology has recently become an alternative to conventional pap staining (CPS) (Nandini, 2012) in the detection of intraepithelial lesions as well as in invasive carcinoma of the uterine cervix. Manual Liquid Based Cytology (MLBC) is a technique that enables the cells to be suspended in a monolayer (Kavatkar et

${ }^{1}$ Department of Histopathology, Institute of Basic Medical Sciences, Khyber Medical University, ${ }^{2}$ Department of Pathology, Institute of Kidney Diseases, Hayatabad Medical Complex, Peshawar, Khyber Pakhtunkhwa, Pakistan *For correspondence: walayats. ibms@kmu.edu.pk 


\section{Shehla Akbar et al}

al., 2008) and can effectively improve cervical cancer screening in a population (Baker, 2002) by enhanced detection of neoplastic and pre-neoplastic disease that can be confirmed histologically and also improves specimen adequacy. This new method was introduced by Maksem et al. (2001) and could possibly reduce the problems associated with CPS. In a recently done comparative study (Moosa et al., 2014), it was concluded that due to a more well preserved cellular structure in MLBC slides as compared to CPS, a more clear picture of cellularity with a clearer background could be seen due to a marked decrease in artifacts, contaminating mucus and blood and lesser degree of cellular overlapping and thus improving diagnosis by improving the quality of slides. Another benefit of liquid based techniques is that, HPV DNA can be tested in the available left over sample from cytology (ACOG Committee, 2009), if the cytological interpretation is abnormal. However, despite all the success of cytology testing for cervical cancer or its precursor lesions, it is essentially prone to variation in a lot of aspects (ACOG Committee, 2009), most important of which is falsenegative results, either due to failure of the pathologist to recognize dysplastic cells or failure to transfer neoplastic or abnormal cells on the slide. Disagreement among different cytopathologists is another factor responsible.

In some studies (Schiffmann et al., 2001) when 5000 cytology samples were subjected to a repeat review by a group of quality-control pathologists, they declared that almost $40 \%$ of the samples which were initially reported as atypical squamous cells of unknown significance (ASCUS), were in fact, negative for any dysplasia on further review while more than $50 \%$ of those initially reported to be high-grade squamous intraepithelial lesion (HSIL) were further declared ASCUS, negative and lowgrade squamous intraepithelial lesion (LSIL). Hence, Pap smear cytology cannot be adequately used as the only screening technique (Sikon et al., 2011) under all circumstances. However, because of its high specificity, when, combined with HPV testing in screening, it gives an advantage.

Most commonly, well-established assays based on polymerase chain reaction (PCR), are carried out for detecting and typing HPV (Tabrizi and Garland, 2006), which are continuously being improved to increase the sensitivity and specificity for detecting HPV infection. These PCR-based assays are dependent on DNA-extract of cells from cervical scrapes or biopsy samples for the detection of HPV.

This study was conducted to assess the frequency of HPV infection in females undergoing Pap smear examination for different gynecological problems using manual liquid based cytology approach.

\section{Materials and Methods}

A total of 200 female patients with gynecological problems were enrolled consecutively in the study. A specifically designed proforma was filled from patients prior to sample collection. Written consents were obtained from the patients and the concerned department and ethical committee. Sample collection was done between August
2012 and October 2013.

Married women of age 21 years to 65 years, who came to the gynecologic OPD for reasons like abnormal uterine bleeding, abnormal vaginal discharge, pelvic or lower abdominal pain, dyspareunia, post-coital bleeding, irregular menstrual cycles, were included in the study.

Married women below 21 years and above 65 years, those having menstrual bleeding at the time of sample collection, those with total abdominal hysterectomy, visible polyps which could bleed readily, drug history of oral contraceptives, radiotherapy, use of vaginal creams, disinfectants and lubricants within the previous 24 hours, severe genital atrophy, pregnancy and women who had a pap test with in the previous three months were excluded from the study.

Cervical scrapings were obtained from the cervix near the squamo-columnar junction with a cytobrush and the tips were snapped-off into a $50 \mathrm{ml}$ sterile plastic bottle containing (Martinez-Contreras et al., 2008) $14 \mathrm{mls}$ PBSEDTA. Once detached from the cytobrush and suspended in the PBSEDTA medium (137 mM sodium chloride $(\mathrm{NaCl}), 2.7 \mathrm{mM}$ potassium chloride $(\mathrm{KCl}), 10 \mathrm{mM}$ disodium phosphate (Na2HPO4), $2 \mathrm{mM}$ di-potassium hydrogen phosphate (KH2HPO2), $25 \mathrm{mM}$ disodium EDTA), (pH 7.4), each sample was fixed by addition of $2 \mathrm{ml} 96 \%$ ethanol. The collected samples placed in a bottle of preservative, were sealed, labeled and the specimen taken to the pathology laboratory at the Institute of Basic Medical Sciences (IBMS) in solution, stored and processed for cytological examination and DNA extraction.

In the laboratory, cell suspensions were mixed with the help of a vortexer and $11 \mathrm{ml}$ of it was transferred into a $15 \mathrm{ml}$ round bottom falcon tube $1.5 \mathrm{ml}$ of alcoholic agar solution (Roose et al., 2001) was added to it. Centrifugation of the falcon tubes was done for 15 minutes at $2000 \mathrm{rpm}$. Cervical cells were trapped in a $1 \mathrm{ml}$ volume of spontaneously produced agar-gel at the bottom of the test tube. The overlying solution was discarded by pouring. Vortex-mixing the agar-gel portion at the bottom caused a rapid shift from gel to solution form, resulting in a viscous cell suspension. With the help of an adjustable jester, $200 \mu 1$ of the cell suspension was taken up and applied drop wise to glass slides, and in this way cytology slides were made. The slides were air-dried and stained using Papanicolaou stains, which were then interpreted by a histopathologist for cytology.

\section{DNA extraction}

From the remaining cell suspension, $250 \mu 1$ was taken for DNA extraction. DNA was extracted using Genomic DNA mini kit (invitrogen by life technologies, USA) following strictly the manufacturer's instructions. The extracted DNA was run on $1 \%$ agarose gel to confirm that enough DNA has been extracted and to assess the quality of the genomic DNA extracted.

The integrity of extracted DNA was checked by PCR using primer set against a segment of $\beta$-globin gene used as an internal-control. The samples were then subjected to a generic screen for the presence of HPVDNA using GP5+/6+ primers which target the conserved sequences 
in the HPV L1 region and can detect a number of low and high risk mucosal HPVs. Next type specific primers were used to detect the presence of HPV-16 and HPV-18 DNA (the most commonly found HR-HPVs in cervical cancer) in the samples. All the primers were selected from previous published work (Romero-Pastrana, 2012) and shown in Table 1.

PCR was carried out in a $25 \mu 1$ reaction volume containing $2 \mu \mathrm{l}$ genomic DNA, $1 \mu \mathrm{l}(0.5 \mu \mathrm{l}$ each $)$ of the forward and reverse primers, $2.5 \mu 1$ of PCR buffer, $1 \mu \mathrm{l}$ of $\mathrm{MgCl} 2,1 \mu 1$ of dNTP mix, $1 \mu 1$ of Taq DNA polymerase and $16.5 \mu \mathrm{l}$ of sterile PCR water. All the above products are manufactured by Life technologies, USA, under the label of invitrogen. PCR was carried out in MultiGeneoptimax by Labnet International. The Cycling conditions were kept as follows: 5 min of denaturation at $94^{\circ} \mathrm{C}$, followed by 35 cycles of 30 seconds of denaturation at $94^{\circ} \mathrm{C}, 30$ seconds of annealing, and 30 seconds of extension at $72^{\circ} \mathrm{C}$. Before the reactions were cooled to room temperature, an additional incubation for 10 minutes at $72^{\circ} \mathrm{C}$ was performed.Annealing temperatures for all four primer pairs were adjusted at optimum temperatures.

$5 \mu \mathrm{l}$ of the PCR product was run on $2 \%$ agarose gel along with a $100 \mathrm{bp}$ molecular weight marker (Invitrogen 100bp DNA Ladder, Life technologies, USA), to estimate the band size of the amplicon. Gel images were analyzed and saved using a major Science Gel Documentation System.

\section{Results}

\section{Cytology findings}

Pap-stained MLBC specimens were screened by histopathologist, according to the Bethesda system 2001 (Moriarty et al., 2002) into normal cells (normal), atypical squamous cells of unknown significance (ASCUS), and cells with low- and high grade intraepithelial lesions (LSILs and HSILs, respectively) and squamous cell carcinoma. The cytological diagnosis was made without knowledge of the PCR results for HPV-DNA. Among 200 clinical samples, 30 samples were reported inadequate either due to absence of endocervical cells, obscured by blood or mucus, less cellularity or some staining problems, 164 were normal out of the rest of the 170 samples which makes around $96.47 \%, 5$ samples were reported to have ASCUS (2.94\%), no samples were reported to have LSIL $(0 \%)$ and 1 sample was reported have HSIL $(0.6 \%)$.

\section{PCR findings}

All the samples were found positive for $\beta$-globin primers, including those reported inadequate on cytology, showing the integrity of the DNA extracted from the cervical cell suspension left over after slide preparations for Pap smear (Figure 1).

Only 4 out of 200 samples (2\%) were found positive for HPV DNA with HPV general primers (Figure 2). On

\section{Table 1. Sequences of Primers Used in the Study}

\begin{tabular}{|c|c|c|c|c|c|}
\hline Genotype & Primer & Primer sequence $5^{\prime}-3^{\prime}$ & Primer Length (No. of bases) & $\operatorname{Tm}{ }^{\circ} \mathrm{C}$ & Amplicon size (bp) \\
\hline HPV 16 & $\mathrm{~F}$ & Ttaggcagcacttggccaacca & 22 & 68 & 207 \\
\hline HPV 16 & $\mathrm{R}$ & Taatccgtcctttgtgtgagct & 22 & 64 & \\
\hline HPV 18 & $\mathrm{~F}$ & Tccgtggtgtgcatcccagcag & 22 & 72 & 274 \\
\hline HPV 18 & $\mathrm{R}$ & Cacttgtgcatcattgtggacc & 22 & 66 & \\
\hline$\beta$-gl & $\mathrm{F}$ & Gaagagccaaggacaggtac & 20 & 62 & 268 \\
\hline$\beta$-gl & $\mathrm{R}$ & Caacttcatccacgttcacc & 20 & 60 & \\
\hline GP & $5+$ & Tttgttactgtggtagatactac & 23 & 62 & $\sim 145$ \\
\hline GP & $6+$ & Gaaaaataaactgtaaatcatattc & 25 & 60 & \\
\hline
\end{tabular}

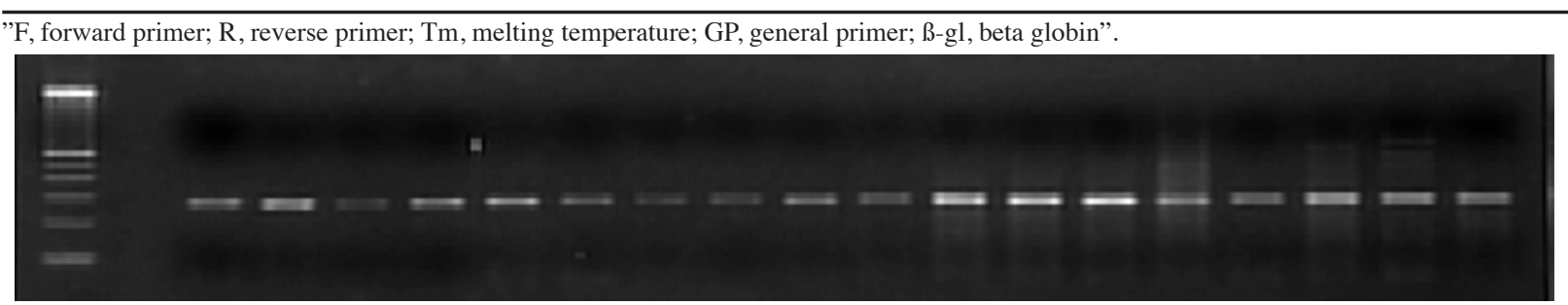

Figure 1. Representative Pictures of PCR Product with Beta Globin Primers. From left to right- position 1 shows $100 \mathrm{bp}$ DNA-ladder for comparison of bands, position 2 is kept empty and position 3 and onwards are bands of beta-globin gene segment which is around 268bp size

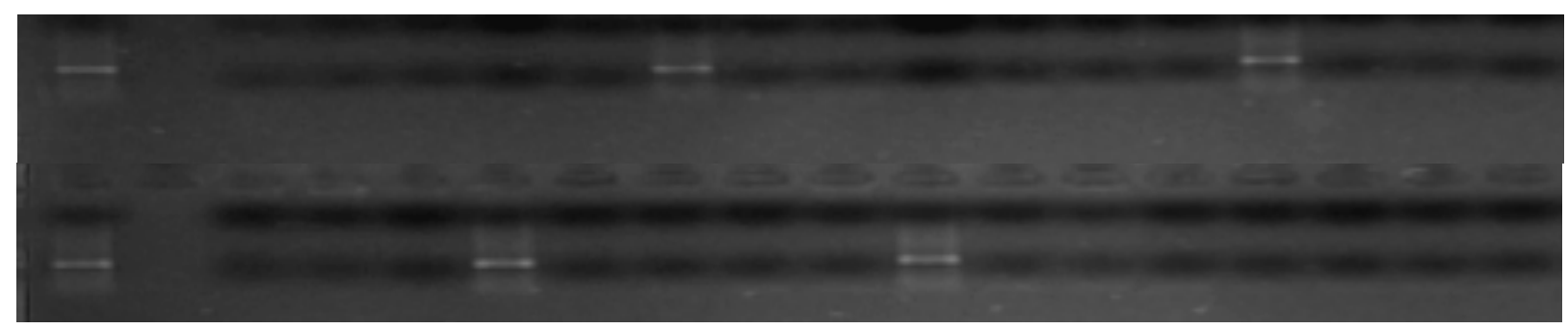

Figure 2. PCR Results for HPV General Primers. Position 1 on the left in each of the upper and lower panel is from known HPV-positive sample used as control for PCR results validation and band size confirmation. The last position on the right in the lower panel is negative control. Two bands in the center of the upper and 2 bands on the lower panel are of the samples found positive on PCR with general primers 


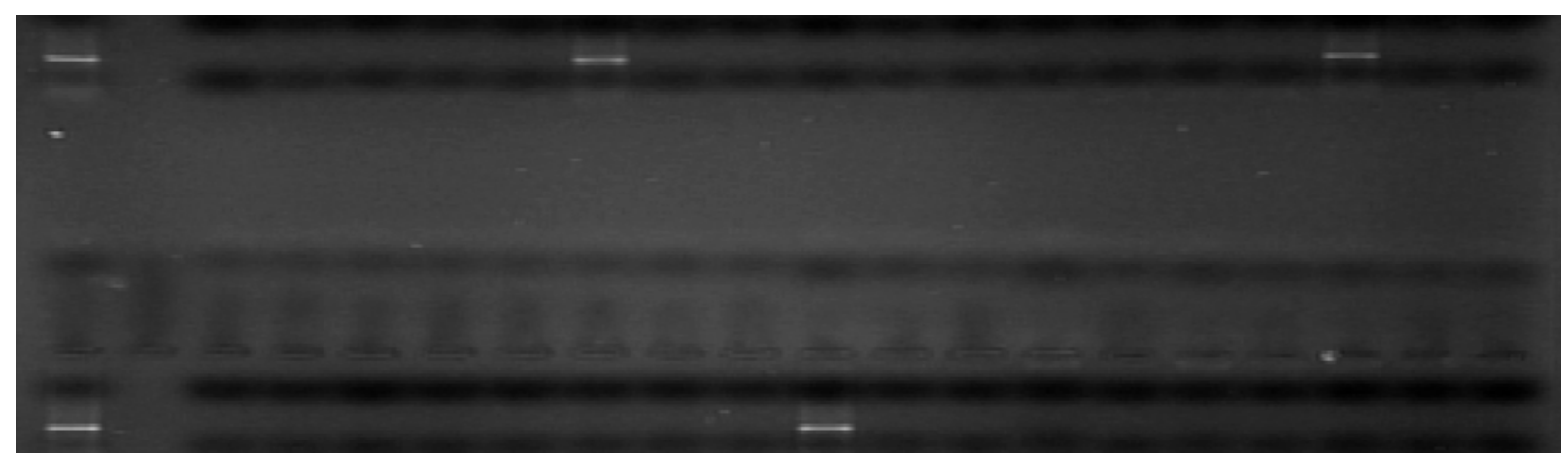

Figure 3. PCR Results for HPV 16 Type Specific Primers. Position 1 on each of the upper and lower panel represents a known HPV 16-positive sample used as control for PCR results validation and band size confirmation which is 207bp. Bands on positions 8 and18 on the upper panel and position 11 on the lower panel are of the samples found positive for HPV 16 type specific primers

type specific PCR $3(1.5 \%)$ samples were found positive for HPV 16 (Figure 3), and $1(0.5 \%)$ sample for HPV 18, making HPV 16 to 18 infection ratio as 3:1 in the general population sampled for this study.

We further compared the cytology findings with PCR results and it was found that out of 5 samples reported to have ASCUS, 1 sample was positive for HPV 18 DNA and 1 for HPV 16 DNA and the rest of the 3 were negative for both HPV general primers as well as type specific primers. The 1 sample reported as HSIL on cytology was HPV 16 positive on PCR. Out of the rest of the normal samples on cytology, only 1 sample was found HPV 16 positive on PCR. The ages of all these HPV positive patients were between 35-45 years except the one patient with HSIL who was a 60 year old lady.

\section{Discussion}

Cervical cancer holds second position in the list of most frequently occurring cancers amongst women throughout the world and as compared to the developed countries, most of it occurs in developing countries (Ferlay et al., 2005; GLOBOCAN, 2012) for which the main reason is shown to be the failure either to begin or then continue successful cervical-cancer screening programs (Wright and Kuhn, 2012). As for screening tests, cytology in association with strong healthcare facilities, has considerably aided in the prevention of cervical cancer in countries that have enough resources to set up and maintain well-conducted programs (Wright and Kuhn, 2012).

The mortality rate of cervical cancer in Pakistan is higher than the incidence rate as compared to various western countries. This elevated mortality rate is assigned to the delayed presentation of cases to the medical facility in Pakistan (Badar et al., 2007) where above $70 \%$ of cancer cases come to seek medical help when the disease has moved to a higher stage (Bhurgri, 2004).

From the facts gathered so far from the very few research projects on human papillomavirus and cervical cancer in Peshawar (Khattak et al., 2006), it seems that still the exact statistical data on the incidence or prevalence of the disease in this population is not known. Health care facilities happen to be insufficiently supplied and the existing ones are inclined to focus more on the curative aspect, rather than on the preventive aspect of diseases and health problems. Women are seldom informed about prevention and advancements such as screening for early detection of cervical cancer; consequently, there is no demand from the female population and no genuine political motivation to set up screening programs. Another major reason seems to be extensive poverty.

Regarding the prevalence of HPV, there are barely any collected figures on the viral presence in the noncancerous specimens from the general female population of Peshawar. Substantiate research that would support in beginning an immunization program against HPV in this kind of population is undoubtedly deficient. From our results it can be seen that HPV do occur in this part of the country, and among the general population the most prevalent type of HPV is 16 and HPV 18. HPV was most frequent in age group 35-45 years old. The most disappointing fact is that the general population including the very highly educated women does not have the knowledge about cervical cancer and its etiological factor plus the health professionals are least interested in educating them about such issues. The gynecologist here relies only on cytology for all kind of patients and if it is negative, the patient is considered negative. From our results we have seen that cytology is prone to falsenegative results and for such women who come to see a doctor only when they become symptomatic, this is a dangerous situation for if the women is actually positive for HPV, it cannot be said that the next time when she visits a doctor what stage of the disease she might be in.

Sherman et al. (2002) suggested that about $15 \%$ of women in annual screening programs who concurrently have a negative Pap test and a positive oncogenic HPV test will have a subsequent abnormal Pap test within 5 years. All these problems could be solved by initiating a proper screening program throughout the country. From our results we have conclude that PCR-based tests are the best for all these cases if conducted at a very basic health facility by a trained medical staff and only the positive cases should be referred to a tertiary care set up to be aided by cytology and further keeping in mind that most of the HPV infections in women below 30 years of age are cleared spontaneously so in this way giving the patient some time to clear the infection as well as closely following her for it through repeat cytology/colposcopy. In addition most of the samples giving ASCUS on cytology results belong to women in the age group above 30 years 
further explaining the need to start screening for this age group. Although many studies supported the use of HPV DNA testing for primary screening largely due to a higher sensitivity, the data nevertheless, suggested that the specificity and positive predictive values might be lower when compared with cytology-based assays (Junyangdikul et al., 2013; Lui, 2013; Priebe, 2013; Siriaunkgul et al., 2014).

In different studies carried out at different institutions of Pakistan, the most frequent explanation for a low Pap test coverage is either lack of knowledge regarding it, or regarding its usefulness, instead of economic limitations or accessibility (Bokutz, 2008). We also noted that patient's information level regarding cervical cancer was very low and literally not a single patient knew about cervical cancer etiology and immunization for its prevention or the mode of transmission of the causative agent. VImproving awareness of the general population on the subject of cervical cancer and its screening is a significant step in improving the coverage of Pap test amongst the women of Peshawar because any harmonized cancer screening attempts are doubtful to be successful without any wellgrounded knowledge amongst the targeted population concerning its aims and effectiveness.

\section{References}

ACOG Committee on Practice Bulletins-Gynecology (2009). ACOG practice bulletin no. 109: cervical cytology screening. Obstet Gynecol, 114, 1409-20.

Badar F, Meerza F, Sultan F, et al (2007). Cervical carcinoma in a muslim community. Asian Pac J Cancer Prev, 8, 24-6.

Baker JJ (2002). Conventional and liquid based cervicovaginal cytology. A comparison study with clinical and histologic Follow-up. Diagn Cytopathology, 27, 185-8.

Belsheim BL, Maksem JA, Finnomore M, et al (2001). Manual method for liquid based cytology. A demonstration using 1,000 Gynaecological cytologies collected directly to vial and prepared by a smear-slide technique. Diagn Cytopathology, 25, 334-8.

Bhurgri Y (2004). Karachi cancer registry data-implications for the national cancer control programme in Pakistan. Asian Pac J Cancer Prev, 5, 77-82.

Bokutz M, Nusrat R, Mazahir S, et al (2008). Pakistani urban population demonstrates a poor knowledge about cancers: A pilot survey. $J$ Cancer Educ, 23, 264-6.

Chaudhry S, Fink A, Gelberg L, et al (2003). Utilization of pap smears by south Asian women living in the United States. $J$ Gen Int Med, 18, 377-84.

Dunne EF, Markowitz LE (2006). Genital human papillomavirus infection. Clin Infectious Diseases, 43, 624-9.

Ferlay J, Parkin DM, Bray F, et al (2005). Global Cancer Statistics 2002. CA: A Cancer J Clin, 55, 74-108.

Garnett GP, Hughes JP, Koutsky L (2002). The theoretical population-level impact of a prophylactic human papillomavirus vaccine. Epidemiology, 13, 631-9.

GLOBOCAN (2012). Estimated cancer incidence, mortality and prevalence worldwide in 2012.

Junyangdikul P, Tanchotsrinon W, Chansaenroj J, et al (2013). Clinical prediction based on HPV DNA testing by hybrid capture 2 (HC2) in combination with liquid-based cytology (LBC). Asian Pac J Cancer Prev., 14, 903-7.

Kavatkar AN, Nagwamshi CA, Dabaks M (2008). Study of a manual method of liquid based cervical cytology. Indian $J$
Pathology Microbiology, 59, 190-4.

Khattak I, Khattak ST, Naheed T, et al (2006). Detection of abnormal cervical cytology by pap smears. Gomal J Med Sci, 4, 74-7.

Koutsky L (1997). Epidemiology of genital human papillomavirus infection. Am J Med, 102, 3-8.

Lui R (2013). Clinical utility of HPV testing. Clin Obstet Gynecol, 56, 17-24.

Martinez-Contreras AL, Sanchez-Garza M, Lopez-Revilla R (2008). Prevalence of high-risk human papillomavirus types in Mexican women with cervical intraepithelial neoplasia and invasive carcinoma. Infectious Agents Cancer, 3, 3 .

Mathers C, Forman D, Parkin DM et al (2010). Estimates of worldwide burden of cancer in: GLOBOCAN 2008. Int $J$ Cancer, 127, 2893-917.

Moosa NY, Khattak N, Alam MI, et al (2014). Comparison of cervical cell morphology using two different cytology techniques for early detection of pre-cancerous lesions. Asian Pac J Cancer Prev, 15, 975-81.

Moriarty A, Davey D, O'Connor D, et al (2002). Forum group members; bethesda 2001 workshop. the 2001 bethesda system: terminology for reporting results of cervical cytology. JAMA, 287, 2114-19.

Nandini NM, Pallavi P, Nandish SM, et al (2012). Manual liquid based cytology in primary screening for cervical cancer-a cost effective preposition for scarce resource settings. Asian Pac J Cancer Prev, 13, 3645-51.

Priebe AM (2013). 2012 cervical cancer screening guidelines and the future role of HPV testing. Clin Obstet Gynecol, 56, 44-50.

Romero-Pastrana F (2012). Detection and typing of human papilloma virus by multiplex PCR with type-specific primers. ISRN Microbiol, 186915.

Schiffman M (2007). Integration of human papillomavirus vaccination, cytology, and human papillomavirus testing. Cancer, 111, 145-53.

Schiffman M, Stoler MH (2001). Atypical squamous cells of undetermined significance-low-grade squamous intraepithelial lesion triage study (ALTS) group. Inter observer reproducibility of cervical cytologic and histologic interpretations: realistic estimates from the ASCUS-LSIL Triage Study. JAMA, 285, 1500-5.

Sherman ME, Castle PE, Wacholder S (2002). Absolute risk of a subsequent abnormal pap among oncogenic human papillomavirus DNA-Positive cytologically negative women. Cancer, 95, 2145-51.

Sikon A, Yen-Liberman B, Jin XW (2011). Cervical cancer screening: Less testing, smarter testing. Cleveland Clin J Med, 78, 737-47.

Siriaunkgul S, Settakorn J, Sukpan K, et al (2014). Populationbased cervical cancer screening using high-risk HPV DNA test and liquid-based cytology in northern Thailand. Asian Pac J Cancer Prev, 15, 6837-42.

Tabrizi S, Garland SM (2006). Methods for HPV detection: polymerase Chain reaction assays. Monsonego J (ed): emerging issues on HPV infections: from science to practice. Basel, Karger. 63-72.

Wright TC, Kuhn L, (2012). Alternative approaches to cervical cancer screening for developing countries. Best Pract Res Clin Obstet Gynaecol, 26, 197-208.

ZurHausen H (1999). Papillomaviruses in human cancers. Proc Assoc Am Physicians. 111, 581-7. 